\title{
Validation of the geriatric anxiety inventory in a duloxetine clinical trial for elderly adults with generalized anxiety disorder
}

\author{
Susan G. Ball, ${ }^{1,4}$ Sarah Lipsius ${ }^{2}$ and Rodrigo Escobar ${ }^{3}$ \\ ${ }^{1}$ Eli Lilly and Company, Corporate Center DC 1542, Indianapolis, IN 46285, USA \\ ${ }^{2}$ Biostatistician InVentiv Health Clinical, 1787 Sentry Parkway, Suite 300, Bldg 16, Blue Bell, PA 19422, USA \\ ${ }^{3}$ Eli Lilly and Company DC 1546, Corporate Center, Indianapolis, IN 46285, USA \\ ${ }^{4}$ Indiana University School of Medicine, Indianapolis, IN 46202, USA
}

ABSTRACT

Background: The Generalized Anxiety Inventory (GAI) has been developed for use in the assessment of anxiety symptoms in older adults ( $\geq 65$ years), but previous validation work has not examined the psychometric qualities of the instrument in relation to treatment. The objective of this study was to examine the performance of the GAI for its internal reliability, convergent and divergent validity, and its sensitivity to treatment.

Methods: Elderly patients with generalized anxiety disorder (GAD) participated in a 10-week double-blind study of duloxetine treatment for patients with GAD. Anxiety symptoms were assessed with the Hamilton Anxiety Rating Scale (HAMA), the Hospital Anxiety and Depression Scale (HADS) anxiety and depression subscales, and the GAI. Internal reliability of the GAI was assessed with Cronbach's $\alpha$. Correlations among the HAMA, HADS, and GAI scores were analyzed to determine convergent and divergent validity. Patients were also compared on remission status using recommended cut-off scores for the GAI.

Results: Patients with GAD, who were at least 65 years of age, were randomly assigned to double-blind treatment with either duloxetine $(N=151)$ or placebo $(N=140)$ for 10 weeks acute therapy. The mean change on the GAI was significantly greater with duloxetine compared with placebo treatment $(-8.36$ vs. -4.96 , respectively, $p \leq 0.001$ ). The GAI demonstrated good internal consistency, good convergent and divergent validity, but suggested cut-off values for caseness with the GAI did not correspond to remission status as measured by the HAMA.

Conclusions: Within an elderly patient population with GAD, the GAI demonstrated sound psychometric qualities and sensitivity to change with treatment.

Key words: anxiety, clinical assessment, scales

\section{Introduction}

As one of the most frequent anxiety disorders, the hallmark of GAD is excessive and difficult to control worry that occurs across a number of different content areas, such as interpersonal/family, work, finances, and minor events (Roemer et al., 1997). Not surprisingly, the number of different life changes and stressors associated with aging, such as retirement, financial changes, and health concerns, result in many elderly adults being particularly vulnerable

Correspondence should be addressed to: Susan G. Ball, Ph.D. Principal Clinical Research Scientist, Eli Lilly and Company, Corporate Center DC 1542, Indianapolis, IN 46285, USA. Phone: (317) 292-8735; Fax: (317) 277-2049. Email: ballsg@lilly.com. Received 1 Oct 2014; revision requested 4 Nov 2014; revised version received $23 \mathrm{Feb} 2015$; accepted $24 \mathrm{Feb} 2015$. First published online 30 April 2015 to the development of GAD. In one epidemiological study, among 2,136 elderly adults between the ages of 65 and 96 years, $79 \%$ of the sample reported worrying excessively during the previous month, with $6.3 \%$ meeting the diagnostic criteria for GAD (Golden et al., 2011). Similarly, in the National Comorbidity Replication Survey (NCS-R), GAD was one of the most prevalent psychiatric disorders in 2,575 elderly adults; the 12-month prevalence rate of GAD was $2.0 \%$ among the "young old, ages 55-64 years," $3.2 \%$ among the "mid-old, ages 65-74 years," $1.7 \%$ among the "old-old, ages $75-$ 84 years," and $0.9 \%$ among the "oldest-old, ages greater than 85 years" (Byers et al., 2010).

A number of instruments have been used to assess anxiety symptoms and disorders within the elderly. While clinical trials typically rely on the 
clinician-based instrument, the HAMA (Hamilton, 1959), as a "gold standard" to assess the efficacy of treatment, the length and complexity of this instrument limits its practicality within other types of clinical settings, which often rely on patientreported measures. In a recent review of measures for the assessment of anxiety in the elderly, the authors found that anxiety questionnaires developed with a broader patient age population are often applied to the elderly without additional psychometric validation (Thiessen and Hunsley, 2012). Given that elderly patients may have cognitive and physical conditions that can impact how they respond to a questionnaire, assuming that an instrument's validity applies to the elderly may be questionable without specific examination.

The need to develop a patient-reported instrument to assess anxiety in elderly patients that would incorporate the sensitivity of clinician-rated scales, such as the HAMA, while considering the needs of elderly patients led to the development of the Geriatric Anxiety Inventory (GAI, Pachana et al., 2007). The instrument was developed to be sensitive to considerations pertinent to the elderly patient population, such as brevity, ease of use, and symptom profile. Specifically, the questionnaire simplifies assessment so that patients indicate "agree or disagree" rather than rating severity or frequency of the symptom; there are no reverse scoring items; and the content of the questions focuses primarily on psychological symptoms of anxiety as opposed to somatic symptoms that may occur as from medical or medicine-related confounds.

Since its introduction, the GAI has been translated into other languages (e.g. MarquezGonzalez, 2012), and has been used to screen for anxiety in elderly patients with medical conditions (e.g. Parkinson's disease, Matheson et al., 2012), and in long-term care (Gerolimatos et al., 2013). The initial psychometric validation demonstrated reliability and sensitivity for the assessment of anxiety among elderly patients using a psychogeriatric service. Despite its promising characteristics, the properties of the GAI have not been studied for its sensitivity to change in a controlled treatment setting. Therefore, the GAI was included as an exploratory objective in a placebo-controlled clinical trial that studied the efficacy of duloxetine (a serotonergic noradrenergic reuptake inhibitor) compared with placebo in older adults ( $\geq 65$ years of age) who were diagnosed with GAD. Results from this trial showed that duloxetine was an efficacious treatment, and details of the trial have been published elsewhere (Alaka et al., 2014). Using the GAI data from this clinical trial, additional psychometric validation for the instrument was undertaken and reported here.

\section{Methods and materials}

The study was conducted in 47 sites across 9 countries that included Argentina, Austria, Canada, Germany, Mexico, Poland, Spain, the United Kingdom, and the United States. Institutional Review Boards at each site approved the protocol, which was developed in accordance with the ethical guidelines of Good Clinical Practice and the Declaration of Helsinki. All patients provided written consent after the study was explained, and their questions were answered before any study procedures were initiated. The trial was publically registered at clintrials.gov (identifier: NCT01118780).

\section{Participants}

Male and female outpatients were included if they were at least 65 years of age and met criteria for GAD as defined by the Diagnostic and Statistical Manual of Mental Disorders, Fourth Edition-Text Revised (DSM-IV TR) (American Psychiatric Association, 2000) and assessed by the Mini International Neuropsychiatric Interview (Sheehan et al., 1998). Each patient was required to have GAD with at least moderate illness severity as defined by the following: a Clinical Global Impressions of Severity of Illness (CGI-S) (Guy, 1976) score $\geq 4$; Covi Anxiety Scale (Lipman et al., 1981) score $\geq 9$; no item score $>3$ on the Raskin Depression Scale (RDS) (Raskin et al., 1969); CAS total score > RDS total score; and a Hospital Anxiety and Depression Scale (HADS) (Zigmond and Snaith, 1983) anxiety subscale score $\geq 10$. A patient was also required to have a Mini-Mental State Examination (MMSE) (Folstein et al., 1975) score of $\geq 24$.

The main exclusion criteria were as follows: a primary Axis I psychiatric diagnosis other than GAD; benzodiazepine use in the prior 14 days before randomization; serious risk for harm to self or others; history of alcohol or any psychoactive substance abuse or dependence (as defined in the DSM-IV TR) within the past 6 months; positive urine screen for any substances of abuse; or the presence of a medical condition that could be potentially worsened by duloxetine.

\section{Study design}

The study was a multi-center, randomized, doubleblind, placebo-controlled, flexible-dose, parallel group trial that consisted of three study periods: screening/ washout period (up to 30 days), 10-week 
therapy period; and an optional 2-week taper-off drug period. Study visits were conducted at weeks $2,4,7$, and 10. Patients were randomly assigned to receive treatment with duloxetine or placebo once daily (QD) in a 1:1 ratio. For the duloxetine group, treatment was initiated with $30 \mathrm{mg}$ QD for at least two weeks. After these two weeks, the dose could be increased by $30-\mathrm{mg}$ increments up to a maximum dose of $120 \mathrm{mg}$. Dose adjustments were based on the investigator's judgment and the degree of clinical improvement. All patients were required to maintain a minimum dose of $30 \mathrm{mg}$ to remain in the treatment phase of the study.

\section{Measurements}

\section{GERIATRIC ANXIETY INVENTORY}

The GAI is a 20 -item self-report measure that uses an agree/disagree response format, with the number of agree responses added into a total score. The content of the majority of items is focused primarily on psychological anxiety, such as "I worry a lot of the time," "I can't help worrying about trivial things," "I think of myself as a worrier," "I miss out on things because I worry too much," although there are a few items that contained somatic references, such as, "I get an upset stomach due to my worrying," "I often feel shaky inside," and "I often feel that I have butterflies in my stomach" (see Pachana et al., 2007 for full item descriptions).

\section{VALIDATION MEASURES}

The primary outcome measure for the study was the clinician-rated HAMA, which was administered using the Structured Interview Guide for the Hamilton Anxiety Rating Scale (Shear et al., 2001). The HAMA total score is the sum of the ratings for each of the 14 items, with item 1 being "anxiety mood" and item 2 being "tension" as the core symptoms of GAD. The HAMA has also been examined based on factor structure, with a HAMA psychic factor consisting of the total of items 1-6 and 14 and a somatic factor consisting of the total of items 7-13. Patients also completed the HADS, which is a 14-item questionnaire that consists of an anxiety subscale (HADS-A) and a depression subscale (HADS-D). Each subscale is the sum of seven questions and the score ranges from 0 to 21 , with higher scores indicating greater severity. Overall improvement during the study was assessed by the clinician using the Clinical Global Impression of Improvement (CGI-I) and by the patient with the Patient Global Impression of Improvement (PGI). Both scales are scored from 1 to 7 with $1=$ "very much better," $4=$ "no change," and $7=$ "very much worse" (Guy, 1976).

\section{Statistical methods}

Psychometric evaluation for the GAI was to assess reliability, convergent and divergent validity, known group validation, and sensitivity to treatment. To assess reliability, an internal consistency estimate was calculated from the GAI individual items using Cronbach's $\alpha$. Convergent validation was assessed by Pearson correlation coefficients between the GAI total score and the HAMA total score, HAMA anxious mood rating (item 1), HAMA tension rating (item 2), HAMA psychic score, and HAMA somatic score. Divergent validation was assessed by the correlation between the GAI and the HADS-D scores at baseline and at endpoint.

The HAMA was also used as an external criterion measure. Patients were assessed as meeting criteria for remission at endpoint if their HAMA total score was $\leq 7$ (Matza et al., 2010). Patients were assessed for their status as a "case" based on the GAI total score, with a GAI total score $<10$ indicating "noncaseness" and GAI total score $\geq 10$ indicating "caseness." The percentage of patients meeting HAMA remission criteria was compared based on their GAI caseness status to determine whether the GAI category could discriminate between patients who were assessed as being in remission.

The GAI sensitivity to change was measured by assessing the mean change in GAI total score from baseline to 10 weeks for patients who had received duloxetine compared with patients who had received placebo. The analysis model was based on a mixed-effects repeated-measures (MMRM) model with terms for treatment, pooled investigative site, visit, treatment-by-visit interaction, baseline GAI total score, and the baseline GAI total score-by-visit interaction. Treatment differences were also analyzed using an ANCOVA model based on the last observation carried forward (LOCF) method with terms for treatment, pooled investigative site, age, and baseline GAI total score. Pearson correlation coefficients were also computed between change in GAI total score across treatments and the CGI-I and PGI-I ratings.

\section{Results}

The majority of patients were female $(77.7 \%)$ and the mean age of the patient population was 71.6 years. Of the 291 patients who were enrolled in the study, 220 completed the study. There were no differences between treatment groups in demographics or baseline illness characteristics (Table 1, data not shown by treatment). Using the baseline GAI item responses, the internal reliability of the GAI was calculated as 0.81 based on Cronbach's $\alpha$. 
Table 1. Patient demographics and baseline illness characteristics

\begin{tabular}{lc}
\hline CHARACTERISTIC & TOTAL $(\mathrm{N}=291)$ \\
\hdashline Age (yrs); mean (SD) & $71.6(5.2)$ \\
\% Female & $77.7 \%$ \\
Race (\%) & \\
$\quad$ Caucasian & $85.6 \%$ \\
African-American & $1.7 \%$ \\
Other & $12 \%$ \\
HAMA total score; mean (SD) & $24.5(6.7)$ \\
HADS anxiety subscale (SD) & $13.7(3.2)$ \\
HADS depression subscale (SD) & $7.4(4.1)$ \\
GAI total score; mean (SD) & $14.3(4.1)$ \\
\hline
\end{tabular}

Abbreviations: SD, standard deviation; HAMA, Hamilton Anxiety Rating Scale; HADS, Hospital Anxiety and Depression Scale; GAI, Geriatric Anxiety Inventory.

\section{Convergent/divergent validation}

Baseline and endpoint scores were used to establish the convergent validation of instruments (Table 2). The GAI total score was correlated with the HAMA total score, factor scores, and individual items as well as the anxiety and depression subscales of the HADS. At both time points, the GAI total score was most highly correlated with the HADS-A subscale scores. Overall, the correlations were higher at endpoint compared with baseline. Between the HADS-A and the HADS-D subscales, the GAI demonstrated a stronger association with the HADS-A subscale. The correlations between the GAI total and the clinician-rated HAMA total scores were lower compared with the GAI and the HADS subscales.

\section{Known groups' validation}

Endpoint scores were examined to determine whether patients met criteria for remission of their GAD based on the HAMA total score. The original discrimination work conducted by Pachana et al. (2007) suggested that a GAI total score of $>10$ represents "caseness" or clinical significance whereas scores $<10$ are indicative of nonclinical cases. The status of remission defined by HAMA total score was then examined by the status of cases by the GAI total score (Figure 1). As can be seen, whereas, scores $\geq 10$ on the GAI were sensitive to cases of nonremission as indicated by the HAMA endpoint score, the results for GAI scores $<10$ were more equivocal. More than half of the patients with lower GAI score were categorized as nonremitted by their endpoint HAMA total score.

\section{Sensitivity to change}

Patients who were treated with duloxetine experienced significantly greater improvement as measured by the GAI total score starting at week 4 and continuing throughout the study ( $p$ ranges from $\leq 0.01$ to 0.001 ) (Figure 2). In both the MMRM and LOCF analyses, the mean difference in improvement for patients who received duloxetine was approximately 3 points greater compared with patients who received placebo. As noted, the correlations between the GAI, HAMA and associated measures, and the HADS were also higher at endpoint, indicating that changes in the GAI were strongly correlated with changes on other disease measures. Similarly, the change in GAI total score also correlated similarly with global assessment of improvement as measured by the clinician, (CGI-I, $r=0.46, p \leq 0.05$ ) and by the patient (PGI-I, $r=0.47, p \leq 0.05$ ).

\section{Discussion}

The results from the current study support the use of the GAI as a measure of severity of anxiety symptoms within an older adult population with GAD. At baseline, given the inclusion criteria, patients were required to have an illness of at least moderate severity, and the mean scores on the HAMA and HADS-A were consistent with this range of severity (Zigmond and Snaith, 1983; Matza et al., 2010). Based on these values, then, a mean GAI score of approximately 14 could be interpreted as also reflecting at least moderate illness severity. From a content validity perspective, the GAI has been shown to have four main factors: central nervous system hyperarousal, excessive worry, difficulty making decisions, and gastrointestinal symptoms (Diefenbach et al., 2014), and good internal consistency, which was also reflected by the strong reliability coefficient within the present trial.

Overall, the GAI demonstrated good convergent validation as shown by its correlations with the total scores as measured by the HADS-A, and to a lesser extent, the HAMA. At baseline, the GAI was more highly correlated with the HADS-A subscale compared with the HAMA, which support that these two instruments were tapping into the same construct and reflect the patient's view of the severity of the anxiety illness. In comparison, the lower correlations with the HAMA total score reflect perhaps different sensitivity of the instrument due to methodology as well as the content. In the HAMA, the rating is based on clinical judgment in which the frequency and severity of symptoms provide a foundation for the scores. In addition, both the HADS-A subscale and the GAI have item content that focus predominantly on psychological symptoms (difficulty concentrating, worrying) as 
Table 2. Correlations of the GAI total score with Hamilton anxiety rating scale and hospital anxiety and depression subscales at baseline and at endpoint

\begin{tabular}{|c|c|c|}
\hline MEASURES & $\begin{array}{l}\text { BASELINE CORRELATION } \\
\text { WITH GAI TOTAL } \\
\text { SCORE }(N=291)\end{array}$ & $\begin{array}{l}\text { ENDPOINT CORRELATION } \\
\text { WITH GAI TOTAL SCORE } \\
(N=275)\end{array}$ \\
\hline HAMA total score & $0.25^{*}$ & $0.55^{*}$ \\
\hline HAMA "anxious mood" item & $0.12^{*}$ & $0.46^{*}$ \\
\hline HAMA "tension" item & $0.12^{*}$ & $0.49^{*}$ \\
\hline HAMA psychic factor score & $0.18^{*}$ & $0.51^{*}$ \\
\hline HAMA somatic factor score & $0.23^{*}$ & $0.50^{*}$ \\
\hline HADS anxiety subscale & $0.51^{*}$ & $0.70^{*}$ \\
\hline HADS depression subscale & $0.28^{*}$ & $0.48^{*}$ \\
\hline
\end{tabular}

Abbreviations: HAMA, Hamilton Anxiety Ratings Scale; HADS, Hospital Anxiety and Depression Scale. ${ }^{*} p \leq 0.05$.

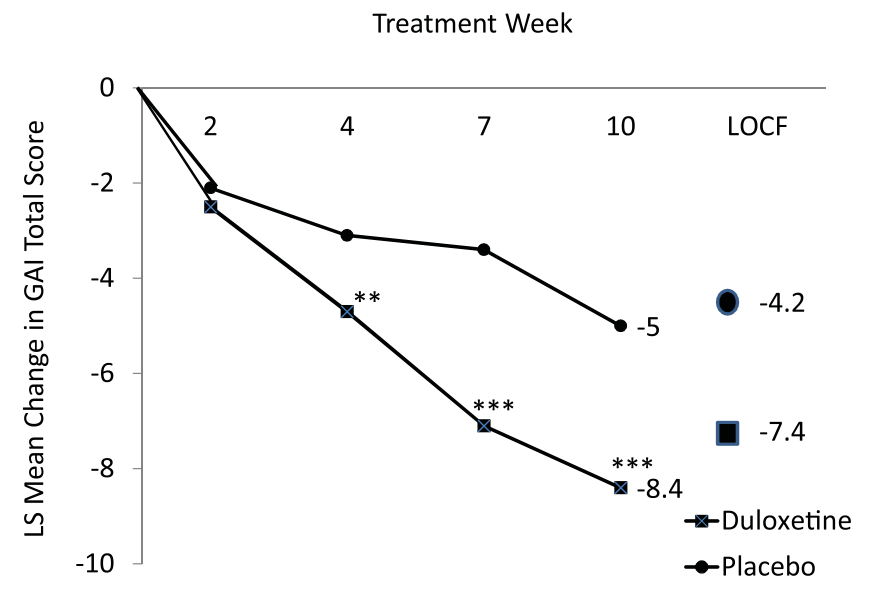

Abbreviations: LS, least squares; ${ }^{*} P \leq .01, * * * P \leq .001$,

Figure 1. (Color online) Least squares mean change in GAI total score from baseline by visit and by last observation carried forward method (LOCF) for patients treated with either duloxetine or placebo.

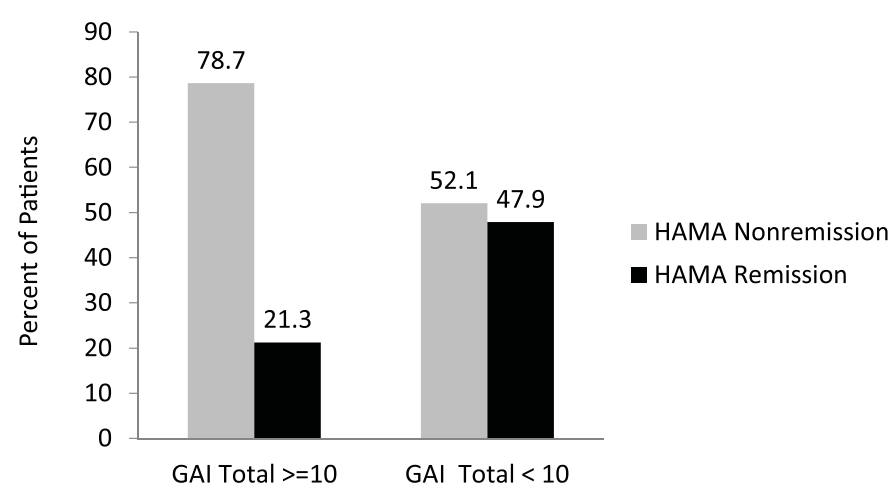

Figure 2. Percent of patients whose HAMA total scores at endpoint met criteria for remission or nonremission by GAI total score "caseness" category at endpoint. Remission for HAMA total score was defined as endpoint score $\leq 7$.

opposed to the mixture of psychic and somatic symptoms in the HAMA scale. At endpoint, the correlations between the GAI and the other anxiety measures were stronger, which may in part be due to less restriction of range in the total scores. At baseline, patients had to have at least moderate severity whereas endpoint scores could range from remitted to severe anxiety. The correlation at endpoint between the GAI and the HADS-A subscale was particularly strong $(r=$ 0.70 ), supporting the convergence between these measures. 
In evaluating the pattern for divergent pattern of correlations among the measures of anxiety and depression, the relationship between GAD and major depressive disorder (MDD) must be considered as these conditions are frequently comorbid, especially among the elderly (Byers et al., 2010). The overlap in these independent but associated constructs can make it difficult to determine the ability of an instrument to distinguish between these illnesses. One strength, however, of the present clinical study is that patients with a diagnosis of MDD were excluded from participation, which is reflected by a mean HADS-D subscale total score that is below the threshold for depression using this measure. Thus, within this more uniform sample, the GAI was able to demonstrate greater divergence between the anxiety and depression construct as measured by its stronger correlation with the HADS-A subscale compared with its correlation with the HADSD subscale. As with the HADS-A subscale, the correlations between the GAI and the HADS-D subscale increased at endpoint reflecting potentially a wider range of depression scores at endpoint despite the initial exclusion criteria.

Since its introduction, the GAI has been primarily studied as a screening instrument within older adults for the purpose of identifying individuals who may need a more thorough evaluation and treatment for an anxiety diagnosis. In the initial validation sample, the suggested cut-off to distinguish between clinical cases and noncases was a GAI total score of 10. More recent work with a sample of older adults receiving home health services supported a score of 9 as the optimal cutoff for sensitivity and specificity in distinguishing between clinical cases (Diefenbach et al., 2009). On the other hand, in a sample of patients with chronic obstructive pulmonary disease, the optimal threshold was 2 or 3, reflecting the overall lower base rate for clinical anxiety within this study (Cheung et al., 2012).

In the present study, the cut-off was validated against the external criterion of remission following treatment as defined by the HAMA clinician rating total score at endpoint. Within this psychiatric population, patients who were identified as "cases" by the GAI were likely cases as identified by the clinician measure of the HAMA; however, approximately half of the "noncases" by the GAI were still symptomatic using the HAMA total score at endpoint. However, as indicated above, one criticism of using the HAMA with older adults is its emphasis on somatic symptoms that may actually be due to other comorbid medical conditions or medications. The HAMA "nonremitted" status could reflect symptoms associated with these confounds. As a screening instrument, the cut-off scores of potential "caseness" will therefore vary according to the targeted patient population, but the sensitivity to change in the GAI scores within the psychiatric population suggests that the instrument may also serve as a useful tool for monitoring the outcome of treatment.

In summary, the GAI represents a brief, patientreported outcome measure that has been designed to assess anxiety symptom severity using a format and structure to enhance its ease of use with the older adult population. As a screening instrument, a number of studies have provided evidence of its utility within populations of particular relevance for the older adult population, such as cognitive impairment (Diefenbach et al., 2013). The present study builds upon this foundation by providing the psychometric characteristics in a well-defined population of older adults with GAD and by demonstrating its usefulness as a measure of change with an established treatment.

\section{Conflict of interest declaration}

Drs. Ball and Escobar are employees and shareholders of Eli Lilly and Company. Dr. Lipsius is an employee of Inventive Health, Inc. Research was funded by Eli Lilly and Company.

\section{Description of authors' roles}

Dr. Ball was involved in the conception and design, acquisition of data, analysis and interpretation of data, drafting of the manuscript, and final approval. Ms. Lipsius contributed by planning in the analysis and interpretation of data, drafting of the manuscript, and final approval. Dr. Escobar contributed in interpretation of data, critical revisions of the manuscript for intellectual content, and final approval.

\section{Acknowledgments}

The authors would like to thank the principal investigators, their staff, and the patients for their participation in this trial.

\section{References}

Alaka, K. J. et al. (2014). Efficacy and safety of duloxetine in the treatment of older adult patients with generalized anxiety disorder: a randomized, double-blind, placebo-controlled trial. International fournal of Geriatric Psychiatry, 29, 978-986. doi: 10.1002/gps.4088 
American Psychiatric Association (2000). Diagnostic and Statistical Manual of Mental Disorders, 4th edn, text rev. Washington, DC: Author.

Byers, A. L., Yaffe, K., Covinsky, K. E., Friedman, M. B. and Bruce, M. L. (2010). High occurrence of mood and anxiety disorders among older adults: the national comorbidity survey replication. Archives of General Psychiatry, 67, 489-496. doi: 10.1001/archgenpsychiatry.2010.35.

Cheung, G., Patrick, C., Sullivan, G., Cooray, M. and Chang, C. L. (2012). Sensitivity and specificity of the geriatric anxiety inventory and the hospital anxiety and depression scale in the detection of anxiety disorders in older people with chronic obstructive pulmonary disease. International Psychogeriatrics, 24, 128-136.

Diefenbach, G. J., Bragdon, L. B. and Blank, K. (2014). Geriatric anxiety inventory: factor structure and associations with cognitive status. American fournal of Geriatric Psychiatry, 22, 1418-1426. doi: 10.1016/j.jagp.2013.04.014.

Diefenbach, G. J., Tolin, D. F., Munier, S. A. and Gilliam, C. M. (2009). Assessment of anxiety in older home care recipients. Gerontologist, 49, 141-153.

Folstein, M. F., Folstein, S. E. and McHugh, P. R. (1975). "Mini-mental state." A practical method for grading the cognitive state of patients for the clinician. Fournal of Psychiatric Research, 12, 189-198.

Gerolimatos, L. A., Gregg, J. J. and Edelstein, B. A. (2013). Assessment of anxiety in long-term care: examination of the geriatric anxiety inventory (GAI) and its short form. International Psychogeriatrics, 25, 1533-1542. doi: $10.1017 /$ S1041610213000847.

Golden, J. et al. (2011). The spectrum of worry in the community-dwelling elderly. Aging and Mental Health, 15, 985-994. doi: 10.1080/13607863.2011.583621.

Guy, W. (1976). The clinical global severity and improvement scale. ECDEU Assessment Manual for Psychopharmacology. US Dept Health, Education and Welfare publication (ADM) 76-338. Rockville, Md: National Institute of Mental Health.

Guy, W. (1976). The patient's global severity and improvement scale. ECDEU Assessment Manual for Psychopharmacology. US Dept Health, Education and Welfare publication (ADM) 76-338. Rockville, Md: National Institute of Mental Health.
Hamilton, M. (1959). The assessment of anxiety states by rating. British fournal of Medical Psychology, 32, 50-55.

Lipman, R. S., Covi, L. and Downing, R. W. (1981). Pharmacotherapy of anxiety and depression: rationale and study design. Psychopharmacology Bulletin, 17, 91-95.

Marquez-Gonzalez, M., Losada, A., Fernandez-Fernandez, V. and Pachana, N. A. (2012). Psychometric properties of the Spanish version of the geriatric anxiety inventory. International Psychogeriatrics, 24, 137-144.

Matheson, S. F. et al. (2012). Validity and reliability of the geriatric anxiety inventory in Parkinson's disease. Australian Fournal of Ageing, 31, 13-16.

Matza, L. S., Morlock, R., Sexton, C., Malley, K. and Feltner, D. (2010). Identifying HAM-A cutoffs for milid, moderate and severe generalized anxiety disorder. International fournal of Methods in Psychiatric Research, 19, 223-232. doi: 10.1002/mpr.323.

Pachana, N. A., Byrne, G. J., Siddle, H., Koloski, N., Harley, E. and Arnold, E. (2007). Development and validation of the geriatric anxiety inventory. International Psychogeriatrics, 19, 103-114.

Raskin, A., Schulterbrandt, J., Reatig, N. and McKeon, J. J. (1969). Replication of factors of psychopathology in interview, ward behavior and self-report ratings of hospitalized depressives, fournal of Nervous and Mental Disease, 148, 8798.

Roemer, L., Molina, S. and Borkovec, T. D. (1997). An investigation of worry content among generally anxious individuals. Fournal of Nervous and Mental Disease, 185, 314-319.

Shear, M. K. et al. (2001). Reliability and validity of a structured interview guide for the Hamilton anxiety rating scale (SIGH-A). Depression and Anxiety, 13, 166-178.

Sheehan, D. V. et al. (1998). The Mini-International Neuropsychiatric Interview (M.I.N.I.): the development and validation of a structured diagnostic psychiatric interview for DSM-IV and ICD-10. Fournal of Clinical Psychiatry, 59(Suppl. 20), 22-33.

Thiessen, Z. and Hunsley, J. (2012). Assessment of anxiety in older adults: a systematic review of commonly used measures. Aging and Mental Health, 16, 1-16.

Zigmond, A. S. and Snaith, R. P. (1983). The hospital anxiety and depression scale. Acta Psychiatrica Scandinavica, 67, 361-370. 